\title{
The Effect of Alfacalcidiol and Metformin on Phenotype Manifestations in Women with Polycystic Ovary Syndrome - a Preliminary Study
}

\author{
I. DRAVECKÁ ${ }^{1}$, J. FIGUROVÁ ${ }^{1}$, M. JAVORSKÝ ${ }^{2}$, J. PETRÍKOVÁ ${ }^{1}$, M. VALKOVÁ ${ }^{1}$, \\ I. LAZÚROVÁ ${ }^{1}$
}

${ }^{1}$ First Department of Internal medicine, Medical faculty of P. J. Šafarik University, Košice, Slovakia, ${ }^{2}$ Fourth Department of Internal medicine, Medical faculty of P. J. Šafarik University, Košice, Slovakia

Received November 23, 2015

Accepted March 24, 2016

On-line July 15, 2016

\section{Summary}

Aim of this study was to evaluate the effect of vitamin D supplementation in obese, insulin resistant and vitamin D deficient PCOS women on biochemical and clinical hyperandrogenism and menstrual irregularity in comparison to effect of metformin or combined metformin plus vitamin $D$ therapy. Thirty nine PCOS women were randomized into three groups and treated with alfacalcidiol (Group 1), combined alfacalcidiol and metformin therapy (Group 2) and metformin (Group 3) for 6 months. Serum TST, fTST, DHEAS, LH and LH/FSH were measured before and after six months of treatment. Menstrual cycle regularity, hirsutism, acne and pregnancy rate were assessed at the same time. There was a significant decrease in TST levels in the Group 2 and slight but not significant decrease in the Group 3. No significant changes in other parameters (fTST, DHEAS, LH, LH/FSH) have been found after 6 months therapy in all three groups. An improvement of menstrual cycle was detected in $78 \%$ of patients in Group 1 $(p<0.04), 80 \%$ in the Group $2(p<0.03)$ and in $90 \%$ in the Group $3(p<0.002)$, respectively. There was no significant improvement of acne and hirsutism in all three groups (all $p$ not significant). Pregnancy rate was higher in the Group 3 as compared with Groups 1 and 2 (67\% vs. $0 \%$ and $25 \%$, respectively), however without statistical significance. Vitamin $D$ administration has no significant effect on androgen levels and clinical features of hyperandrogenism in obese vitamin $D$ deficient PCOS women. However, it can potentiate effect of metformin on testosterone levels and LH/FSH ratio but not on clinical hyperandrogenism and pregnancy rate.

\section{Key words}

Polycystic ovary syndrome - Alfacalcidiol - Metformin • Hyperandrogenism • Menstrual cycle irregularity • Pregnancy rate

\section{Corresponding author}

I. Dravecká, 1st Department of Internal Medicine, Medical Faculty of P. J. Šafarik University, Trieda SNP 1, 04011 Košice, Slovakia. Fax: +421 442343411 . E-mail: ingrid.dravecka@upjs.sk

\section{Introduction}

Polycystic ovary syndrome (PCOS) is the most common endocrine disease affecting up to $18 \%$ women of reproductive age (March et al. 2010). It is characterized by biochemical and/or clinical hyperandrogenism presented as hyperandrogenemia and/or hirsutism, acne and androgenic alopecia, ovulatory dysfunction resulting in oligo - and/or anovulation and the presence of polycystic ovaries on ultrasound (Azziz et al. 2006). PCOS is also very often associated with multiple metabolic abnormalities such as insulin resistance (IR), hyperinsulinemia, dyslipidemia and central obesity, which are not included in diagnostic criteria, but their presence increases the risk of developing type 2 diabetes mellitus and cardiovascular morbidity and mortality (Diamanti-Kandarakis and Dunaif 2012, Hahn et al. 2006).

PCOS is still surrounded by considerable uncertainty in etiology and pathogenesis limiting possibilities of a causal therapy. Vitamin D deficiency is 
very common in PCOS women and few observational studies demonstrated that low vitamin D levels were associated with insulin resistance, obesity, hirsutism and also hyperandrogenism resulting in increased cardiovascular risk in affected women (Wehr et al. 2009, Yildizhan et al. 2009, Li et al. 2011, Figurová et al. 2015). Prevalence of vitamin D deficiency is very high reaching in some studies up to $70 \%$ of all women in reproductive age (Yildizhan et al. 2009, Figurová et al. 2015, Kotsa et al. 2009). Because of its common association with metabolic syndrome which is more prevalent in PCOS women, there is still discussion of the impact of vitamin D supplementation on various metabolic and phenotypic manifestations in PCOS. The role of vitamin D and calcium supplementation on various features of PCOS has been studied in several studies and their effectiveness was described mainly due to their effect on body weight and follicular maturation. However, only few interventional studies documented an improvement of insulin sensitivity and metabolic abnormalities after vitamin D supplementation in PCOS women (Kotsa et al. 2009, Wehr et al. 2011). Some of them also demonstrated an improvement of clinical hyperandrogenism, ovulation and fertility (Selimoglu et al. 2010, Thys-Jacobs et al. 1999, Rashidi et al. 2009). Nevertheless, there are still controversies concerning effects of vitamin D replacement therapy on various phenotypic manifestations of PCOS, especially clinical features of hyperandrogenism and hyperandrogenemia.

The aim of this study was to assess the effect of vitamin D (alphacalcidiol) supplementation on phenotype manifestations of PCOS, i.e. serum androgen levels, clinical hyperandrogenism (hirsutism and acne), menstrual cycle regulartity and pregnancy rates in vitamin D deficient insulin resistant PCOS women in comparison with the effect of metformin and combined metformin plus vitamin D therapy.

\section{Methods}

\section{Subjects and methods}

The study group consisted of 39 PCOS women of reproductive age primary identified in an outpatient Department of gynaecology, endocrinology and diabetology at University Hospital of Louis Pasteur, Košice, Slovakia and consequently examined in the $1^{\text {st }}$ Internal Medicine Department over a period between September 2010 - March 2013. 39 PCOS patients fulfilling inclusion criteria were divided into
3 therapeutic groups using a random number table, each containing 13 women. The average age of PCOS women was in the Group $129.3 \pm 4.89$ years, in Group 2 $29.2 \pm 5.42$ years and in Group $327.6 \pm 4.96$ years. After collecting baseline blood samples, all PCOS women in Group 1 received $1 \mu \mathrm{g}$ alfacalcidiol per day, orally (ALPHA D3 $1 \mu \mathrm{g}$ cps, TEVA Pharmaceuticals Slovakia s.r.o., SR), PCOS women in Group 2 received combined therapy $1 \mu \mathrm{g}$ of alfacalcidiol (ALPHA D3 $1 \mu \mathrm{g}$ cps, TEVA Pharmaceuticals Slovakia s.r.o., SR) and metformin in dose 1,700-2,550 $\mathrm{mg}$ daily (Siofor $850 \mathrm{mg}$ tbl, Berlin Chemie AG, Berlin) and PCOS women in Group 3 used metformin 1,700-2,550 mg per day, orally (Siofor $850 \mathrm{mg}$ tbl, Berlin Chemie AG, Berlin) for 6 months. Study design and number of the patients in each therapeutic arm who finished the study and was included to the final statistical analysis is shown in flow chart (Fig. 1).

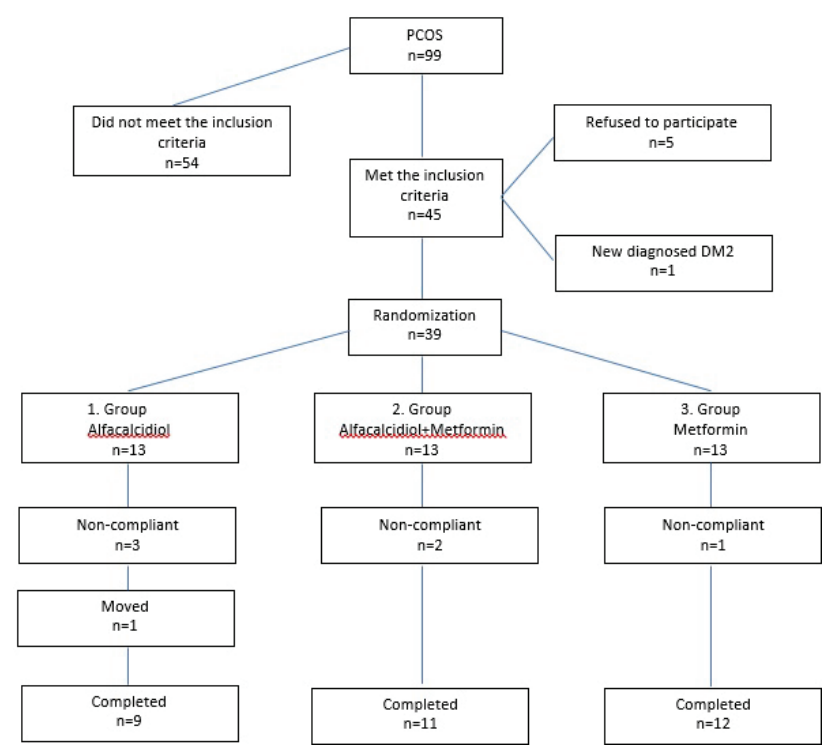

Fig. 1. Flow chart of study design.

The diagnosis of PCOS was based on the Androgen Excess Society criteria (Azziz et al. 2006). Hyperandrogenism was determined by the clinical presence of hirsutism (modified Ferriman-Gallwey score $\geq 6$ ), acne or alopecia and/or elevated androgen levels. Other common causes of hyperandrogenism and/or anovulation (Cushing's syndrome, congenital adrenal hyperplasia, androgen-secreting tumors, hyperprolactinemia, thyroid dysfunction) were ruled out by careful endocrine examination. Additional inclusion criteria comprised presence of vitamin D deficiency, which was defined as serum levels of $25(\mathrm{OH}) \mathrm{D}<30 \mathrm{ng} / \mathrm{ml}$ (Bouillon et al. 2013), HOMA-IR $>2.5$. 
All study participants did not use oral contraceptives, insulin sensitisers, hypolipidemic agents and also any medication known to affect endocrine parameters, carbohydrate or lipid metabolism during last 3 months. All women were advised to maintain their usual dietary habits, not to modificate other lifestyle factors such as sunlight exposure and physical activity during the study that could affect the levels of vitamin D and metabolic profile.

Written informed consent was obtained from each study participant. The study design was approved by the local ethics committee and fulfilled the ethical guidelines of the most recent declaration of Helsinki.

The clinical evaluation consisted of a detailed medical and menstrual history and physical examination. Standard anthropometric data were obtained from each subject (height, weight, waist circumference, body mass index (BMI). The regularity of menstrual cycle ssessed according to time intervals (21-35 days), hirsutism according to modified Ferriman - Galway score $\geq 6$, presence of acne as well as pregnancy rate were compared between all three groups. Blood samples for selected endocrine parameters (total testosterone (TST), freetestosterone (fTST), dehydroepiandrosterone sulphate (DHEAS), sexual-hormone binding globuline (SHBG), free androgen index (FAI), luteinizing hormone (LH), follicle-stimulating hormone (FSH), LH/FSH ratio, $25(\mathrm{OH}) \mathrm{D})$ were taken before and after 6 months of the treatment.

\section{Biochemical analysis}

Serum 25(OH)D levels were measured using a commercially available radioimmunoassay kit (IDS Ltd.) with intra- and inter- assay coefficients of variation $(\mathrm{CV})$ of 5.0 vs $7.3 \%$ respectively. Serum levels of $\mathrm{LH}, \mathrm{FSH}$, testosterone, DHEAS, SHBG were determined using Chemiluminescent Microparticle Immunoassay (analyser Architect, module C). Free testosterone was detected by Radioimmunoassay using commercially available kits of DIA Source.

\section{Statistical analysis}

Data are presented as mean \pm standard deviation or as count (percentages), unless stated otherwise. Comparing the changes of variables between 2 periods of intervention within therapeutic group was realised by pair T-test in continual variables and by Wilcoxon test in categorical variables. Assessment the significance of differences in effect of therapeutic intervention between therapeutic groups was tested by parametric ANOVA test and nonparametric Kruskall Wallis test. p values of $<0.05$ were considered statistically significant. Statistical analyses were performed with SPSS software version 15 (IBM, USA).

\section{Results}

Anthropometric, metabolic and endocrine data in all therapeutic groups before study are shown in Table 1 . There were no significant differences in mean age, weight and BMI, between all three groups in the beginning of the study (all p not significant). Additionally, all groups did not differ in serum levels of insulin, lipid profile and also in hormonal status including TST and fTST, SHBG, FAI, DHEAS, LH and LH/FSH ratio (all $\mathrm{p}$ not significant) (Table 1).

After 6 months treatment period we observed a significant decrease in serum TST levels in the Group 2 (alfacalcidiol+metformin) and decrease in serum TST with borderline significance in the Group 3 (metformin). There were no significant changes in other androgenes, i.e. fTST, DHEAS and FAI after 6 months period in all three groups (all $\mathrm{p}$ not significant). In addition serum LH levels and LH/FSH ratio did not change significantly after treatment in all groups as well (Table 2).

Changes of serum TST, fTST, LH and LH/FSH after treatment within each group are shown in Figure 2. There was a significant change in LH/FSH ratio after treatment period between all three groups $(p=0.023)$. The most pronounced trend to decrease $\mathrm{LH} / \mathrm{FSH}$ ratio was in the Group 2 (alfacalcidiol+metformin). Further we observed an effect of therapeutical intervention on a decrease in serum fTST levels between all groups with borderline significance $(\mathrm{p}=0.089)$. The most pronounced effect of treatment on a decrease in fTST levels was detected in the Group 3 (metformin) (Fig. 2).

Table 3 summarizes the improvement of clinical features of hyperandrogenism and also pregnancy rates in all three groups. An improvement of menstrual cycle (MC) irregularity was detected in $78 \%$ of patients in the Group 1 (alfacalcidiol) $(\mathrm{p}<0.04), 80 \%$ in the Group 2 (alfacalcidiol+metformin) $(\mathrm{p}<0.03)$ and in $90 \%$ in the Group 3 (metformin) $(\mathrm{p}<0.002)$, respectively. There was no significant effect on the improvement of acne and hirsutism in all three groups (all $\mathrm{p}$ not significant). Pregnancy rate was higher in the Group 3 (metformin) as compared with groups 1 and $2(67 \%$ vs. $0 \%$ and $25 \%$ respectively), however without statistical significance. 
Table 1. Anthropometric, metabolic and endocrine characteristics of therapeutic group.

\begin{tabular}{|c|c|c|c|c|}
\hline Variables $^{\mathrm{a}}$ & $\begin{array}{c}\text { Group } 1 \\
\text { VIT D } \\
(n=9)\end{array}$ & $\begin{array}{c}\text { Group } 2 \\
\text { VIT D+MET } \\
(\mathbf{n}=\mathbf{1 1})\end{array}$ & $\begin{array}{c}\text { Group } 3 \\
\text { MET } \\
(n=12)\end{array}$ & $\mathbf{p}$ \\
\hline Age (years) & $29.33 \pm 4.89$ & $29.2 \pm 5.42$ & $27.6 \pm 4.96$ & NS \\
\hline Weight (kg) & $87.87 \pm 20.15$ & $92.10 \pm 20.07$ & $84.13 \pm 12.13$ & NS \\
\hline Weight circumference (cm) & $96.33 \pm 13.77$ & $106.87 \pm 16.40$ & $93.67 \pm 13.75$ & 0.052 \\
\hline$B M I\left(\mathrm{~kg} / \mathrm{m}^{2}\right)$ & $32.24 \pm 6.71$ & $33.15 \pm 7.11$ & $29.85 \pm 5.43$ & 0.395 \\
\hline Glucose $_{0}(\mathrm{mmol} / \mathrm{l})$ & $4.94 \pm 0.49$ & $4.85 \pm 0.45$ & $4.97 \pm 0.73$ & 0.941 \\
\hline $\operatorname{Insulin}_{0}(\mu I U / m l)$ & $16.82 \pm 5.76$ & $22.29 \pm 13.19$ & $18.63 \pm 11.68$ & 0.346 \\
\hline$H O M A-I R$ & $3.74 \pm 1.58$ & $4.88 \pm 3.24$ & $4.25 \pm 3.28$ & 0.359 \\
\hline Total cholesterol (mmol/l) & $5.17 \pm 1.06$ & $4.97 \pm 0.80$ & $5.16 \pm 0.99$ & 0.896 \\
\hline Triglycerides $(\mathrm{mmol} / \mathrm{l})$ & $1.42 \pm 0.73$ & $1.84 \pm 1.31$ & $1.39 \pm 0.6$ & 0.464 \\
\hline Testosterone $(\mathrm{ng} / \mathrm{ml})$ & $1.19 \pm 0.3$ & $1.15 \pm 0.43$ & $1.12 \pm 0.39$ & 0.648 \\
\hline f-Testosterone $(\mathrm{pg} / \mathrm{ml})$ & $4.05 \pm 0.95$ & $4.18 \pm 1.07$ & $4.26 \pm 1.31$ & 0.979 \\
\hline SHBG $($ nmol/l) & $48.9 \pm 30.67$ & $33.41 \pm 17.21$ & $48.93 \pm 29.25$ & 0.120 \\
\hline FAI & $8.6 \pm 5.4$ & $14.6 \pm 11.38$ & $10.78 \pm 5.2$ & 0.109 \\
\hline DHEAS $(\mu g / 100 \mathrm{ml})$ & $339.40 \pm 155.15$ & $329.0 \pm 206.36$ & $388.20 \pm 190.89$ & 0.620 \\
\hline LH/FSH ratio & $1.40 \pm 0.89$ & $1.49 \pm 0.96$ & $1.10 \pm 0.65$ & 0.614 \\
\hline$L H(I U / m l)$ & $7.09 \pm 4.91$ & $9.06 \pm 8.65$ & $7.06 \pm 4.28$ & 0.861 \\
\hline $25(\mathrm{OH}) \mathrm{D}(\mathrm{ng} / \mathrm{ml})$ & $24.1 \pm 8.0$ & $22.0 \pm 8.4$ & $24.3 \pm 6.8$ & 0.339 \\
\hline
\end{tabular}

${ }^{a}$ Data presented as mean \pm SD or percentages, $p \leq 0.05$ is statistically significant.

Table 2. Serum androgen levels, $\mathrm{LH}$ and LH/FSH ratio before and after treatment within each group in vitamin D deficient PCOS women.

\begin{tabular}{|c|c|c|c|c|c|c|c|c|c|}
\hline \multirow[b]{2}{*}{ Variables } & \multicolumn{3}{|c|}{$\begin{array}{c}\text { Group } 1 \\
\text { VIT D } \\
\mathbf{n}=9\end{array}$} & \multicolumn{3}{|c|}{$\begin{array}{c}\text { Group } 2 \\
\text { VIT D+MET } \\
\mathbf{n}=\mathbf{1 1}\end{array}$} & \multicolumn{3}{|c|}{$\begin{array}{c}\text { Group } 3 \\
\text { MET } \\
n=12\end{array}$} \\
\hline & $\begin{array}{l}\text { Before } \\
\mathbf{x} \pm \text { SD }\end{array}$ & $\begin{array}{l}\text { After } \\
\mathbf{x} \pm \mathbf{S D}\end{array}$ & $\mathbf{p}$ & $\begin{array}{l}\text { Before } \\
\mathbf{x} \pm \text { SD }\end{array}$ & $\begin{array}{l}\text { After } \\
\mathbf{x} \pm \text { SD }\end{array}$ & $\mathbf{p}$ & $\begin{array}{c}\text { Before } \\
\mathbf{x} \pm \text { SD }\end{array}$ & $\begin{array}{l}\text { After } \\
\mathbf{x} \pm \text { SD }\end{array}$ & $\mathbf{p}$ \\
\hline $\begin{array}{l}T S T \\
(n g / m l)\end{array}$ & $1.19 \pm 0.30$ & $1.22 \pm 0.30$ & 0.936 & $1.15 \pm 0.43$ & $1.03 \pm 0.46$ & 0.016 & $1.12 \pm 0.39$ & $0.97 \pm 0.33$ & 0.088 \\
\hline $\begin{array}{l}f T S T \\
(p g / m l)\end{array}$ & $4.05 \pm 0.95$ & $4.70 \pm 0.94$ & 0.128 & $4.18 \pm 1.07$ & $4.53 \pm 1.18$ & 0.107 & $4.26 \pm 1.31$ & $3.74 \pm 1.10$ & 0.154 \\
\hline $\begin{array}{l}S H B G \\
(\mathrm{nmol} / \mathrm{l})\end{array}$ & $48.9 \pm 30.67$ & $44.33 \pm 28$ & 0.229 & $33.4 \pm 17.2$ & $28.4 \pm 14.5$ & 0.256 & $48.9 \pm 29$ & $56.3 \pm 49.7$ & 0.654 \\
\hline$F A I$ & $8.6 \pm 5.4$ & $12.39 \pm 8.06$ & 0.213 & $14.6 \pm 11.38$ & $\begin{array}{c}13.27 \pm \\
7.56\end{array}$ & 0.621 & $10.78 \pm 5.2$ & $10.72 \pm 9.38$ & 0.627 \\
\hline $\begin{array}{l}\text { DHEAS } \\
(\mu \mathrm{g} / 100 \mathrm{ml})\end{array}$ & $\begin{array}{l}339.4 \pm \\
155.15\end{array}$ & $\begin{array}{c}431.22 \pm \\
207.92\end{array}$ & 0.499 & $\begin{array}{l}329.0 \pm \\
206.36\end{array}$ & $\begin{array}{c}322.42 \pm \\
184.13\end{array}$ & 0.316 & $\begin{array}{c}388.20 \pm \\
190.89\end{array}$ & $\begin{array}{c}406.50 \pm \\
209.23\end{array}$ & 0.987 \\
\hline $\begin{array}{l}\text { LH/FSH } \\
\text { ratio }\end{array}$ & $1.40 \pm 0.89$ & $1.94 \pm 1.99$ & 0.128 & $1.49 \pm 0.96$ & $1.12 \pm 0.51$ & 0.193 & $1.10 \pm 0.65$ & $2.82 \pm 5.31$ & 0.289 \\
\hline$L H(I U / l)$ & $7.09 \pm 4.91$ & $8.33 \pm 6.42$ & 0.130 & $9.06 \pm 8.65$ & $5.87 \pm 2.90$ & 0.228 & $7.06 \pm 4.28$ & $11.91 \pm 20.41$ & 0.397 \\
\hline $\begin{array}{l}25(\mathrm{OH}) D \\
(n g / m l)\end{array}$ & $24.1 \pm 8.0$ & $22.7 \pm 6.6$ & 0.575 & $22.0 \pm 8.4$ & $23.2 \pm 8.2$ & 0.289 & $24.3 \pm 6.8$ & $23.5 \pm 6.5$ & 0.530 \\
\hline
\end{tabular}

${ }^{a}$ Values are expressed as mean $\pm S D, p \leq 0.05$ is statistically significant. $\Delta$ means change in variables after 6 months treatment. $p$ value indicates statistical significant change in variables after 6 months treatment within the group. 


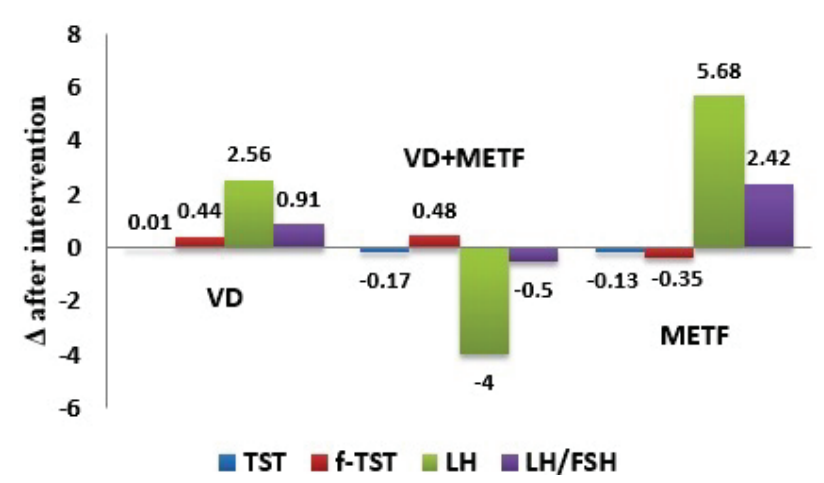

Fig. 2. Changes in serum TST, fTST, LH and LH/FSH levels after treatment within each group of vitamin D deficient PCOS women.

\section{Discussion}

In this study we compared the effect of alfacalcidiol (VIT D), metformin plus alfacalcidiol (VIT D+MET) and metformin (MET) therapy on endocrine profile and phenotypic manifestations (menstrual cycle, hirsutism, acne and pregnancy rate) in vitamin D deficient, insulin resistant PCOS women. We observed a significant decrease in total testosterone levels in the VIT D+MET group after 6 months and slight but not significant decrease in the MET group. No significant changes in other androgens, such as fTST, DHEAS and FAI were detected in all three groups. When assessed hormonal changes after treatment, there was a significant difference in $\Delta \mathrm{LH} / \mathrm{FSH}$ ratio $(\mathrm{p}=0.023)$ between all three groups (we observed the most pronounced decrease in LH/FSH ratio in VIT D + MET group) and slight but not significant difference in fTST decline between groups. The most pronounced effect of treatment on a decrease in fTST was detected in the MET group.

The effect of vitamin D on metabolic status in PCOS women has been evaluated in several studies with relatively small number of patients in randomized groups.
Many of them has had short treatment period and used different doses of vitamin D as well as different types of vitamin D were used. Despite these differences majority of studies did not confirm any changes in metabolic parameters such as BMI, waist circumference and insulin sensitivity, as was also demonstrated by our previous study (Bonakdaran et al. 2012, Ardabili et al. 2012, Figurová et al. 2016). However there are only few studies evaluating the effect of vitamin D on clinical and biochemical hyperandrogenism in PCOS vitamin D deficient women.

The study of Bonakdaran et al. (2012) did not find any changes in testosterone and DHEAS levels after treatment between metformin, calcitriol and placebo groups. This is similar to our results; however their study did not assess the effect of combined therapy with metformin plus vitamin D. They also found a significant impact of calcitriol treatment on ovulation indicating that vitamin $\mathrm{D}$ and calcium may play a role in oocyte maturation. On the other hand vitamin D deficiency causes obesity and insulin resistance inducing hyperandrogenism followed by menstrual irregularity (Bonakdaran et al. 2012).

In the recently published study of Tehrani et al. (2014), that was focused on efficacy of vitamin D on menstrual cycle, BMI and hyperandrogenism in PCOS women, authors compared four groups i.e. metformin, vitamin $\mathrm{D}$, metformin plus vitamin $\mathrm{D}$ and placebo. The results indicated that using metformin with vitamin $\mathrm{D}$ and calcium seems to be more effective for hirsutism, menstrual cycle regularity and ovulation whereas metformin alone had more significant effect on acne (Tehrani et al. 2014). This is in a conflict with our study in which we were not able to demonstrate a significant effect of vitamin $\mathrm{D}$, metformin or vitamin $\mathrm{D}$ plus metformin the improvement of acne and hirsutism.

Table 3. Improvement of $M C$ irregularity, acne, hirsutism and pregnancy rate in all three groups after 6 months of treatment.

\begin{tabular}{|c|c|c|c|c|c|c|}
\hline \multirow[b]{2}{*}{ Variables } & \multicolumn{2}{|c|}{$\begin{array}{c}\text { Group 1 } \\
\text { VIT D } \\
\mathbf{n}=9\end{array}$} & \multicolumn{2}{|c|}{$\begin{array}{c}\text { Group 2 } \\
\text { VIT D+MET } \\
\mathbf{n}=\mathbf{1 1}\end{array}$} & \multicolumn{2}{|l|}{$\begin{array}{c}\text { Group 3 } \\
\text { MET } \\
n=12\end{array}$} \\
\hline & $\begin{array}{c}\text { Improvement } \\
\text { n (\%) }\end{array}$ & $\mathbf{p}$ & $\begin{array}{c}\text { Improvement } \\
\text { n (\%) }\end{array}$ & $\mathbf{p}$ & $\begin{array}{c}\text { Improvement } \\
\text { n (\%) }\end{array}$ & $\mathbf{p}$ \\
\hline Menstrual cycle & 7/9 (77.8 \%) & 0.030 & $8 / 10(80 \%)$ & 0.040 & 9/10 (90\%) & 0.002 \\
\hline Acne & $1 / 4(25 \%)$ & 0.670 & $1 / 2(50 \%)$ & 0.943 & $2 / 6(33.3 \%)$ & 0.867 \\
\hline Hirsutism & $1 / 8(12.5 \%)$ & 0.500 & 3/9 (33.3\%) & 0.795 & $2 / 8(25 \%)$ & 0.170 \\
\hline Pregnancy rate & $0 / 3(0 \%)$ & 0.700 & $1 / 4(25 \%)$ & 0.835 & $2 / 3(66.6 \%)$ & 0.493 \\
\hline
\end{tabular}


Concerning menstrual irregularity the improvement of menstrual cycle was detected in all three groups with statistical significance and was more pronounced in the metformin group. Pregnancy rate was higher in the metformin group as compared with other groups $(67 \%$ vs $0 \%$ and $25 \%$ ), however without a statistical significance. It is partly in agreement with previous studies. In the study of Firouzabadi et al. (2012) authors compared influence of vitamin D and calcium in PCOS women on metabolic and endocrine abnormalities in infertile PCOS women. A better improvement of menstrual cycle irregularity was observed in the group treated with metformin plus vitamin D and calcium as compared to the group of metformin alone (70\% vs. $58 \%$ ). Authors found a positive effect of vitamin D supplementation on follicle maturation and also the improvement of hyperandrogenism (Firouzabadi et al. 2012). This is the same conclusion reported by ThysJacobs et al. (1999) and is similar to our results concerning biochemical hyperandrogenism (total testosterone and LH/FSH ratio changes), but not clinical features (Thys-Jacobs et al. 1999).

To our best knowledge this is the first randomized interventional study assessing the effect of alfacalcidiol on hyperandrogenemia and menstrual cycle abnormalities in PCOS women after 6 months therapy. A major strength of this study is homogeneity of all groups that were age, BMI and IR matched and all of them were vitamin D deficient. Secondly there are only few trials with the 6 months duration of vitamin D supplementation, as was done in our study. On the other hand this study has several limitations. The main limitations are small groups of patients completed the trial and no evaluation of dominant follicle on ultrasound examination after the six months treatment. Further possible limitation is the use of alfacalcidiol during the treatment period which was connected with difficulties to compare its effect on endocrine profile and clinical features of PCOS with other therapeutic regimens.

Similarly to majority of the studies we can conclude that vitamin $\mathrm{D}$ administration has no significant effect on androgen levels and clinical features in obese vitamin D deficient PCOS women. However it can potentiate effect of metformin on testosterone levels and LH/FSH ratio but not on clinical hyperandrogenism and pregnancy rate. To confirm these findings further randomized controlled studies on larger group of PCOS patients are required.

\section{Conclusions}

We compared the effect of vitamin D, metformin plus vitamin $\mathrm{D}$ and metformin therapy on endocrine profile and phenotypic manifestations in vitamin D deficient, insulin resistant PCOS women. We have found out a significant decrease in total testosterone levels in the vitamin $\mathrm{D}+$ metformin group after 6 months and slight but not significant decrease in the metformin group. There were any significant changes in other androgens in all three groups. We have noticed a significant difference in $\triangle \mathrm{LH} / \mathrm{FSH}$ ratio between all three groups and slight but not significant difference in fTST decline between groups. The most pronounced effect of treatment on a decrease in fTST was observed in the metformin group. We have observed an improvement of menstrual cycle irregularity in $78 \%$ of patients in the alfacalcidiol group, in $80 \%$ in the combined group and in $90 \%$ in the metformin group. There was no significant effect on the improvement of acne and hirsutism in all three groups. Pregnancy rate was higher in the metformin group, however, without statistical significance. The limitations of this study are small groups of patients and missing evaluation of a dominant follicle on ultrasound examination after the six months treatment. The next limitation can be the use of alfacalcidiol during the treatment so we could not check the change of the level of vitamin D after treatment.

\section{Conflict of Interest}

There is no conflict of interest.

\section{Acknowledgements}

We thank the study participants whose continued dedication and commitment make this work possible. This work was supported by VEGA grant No 1/0048/11.

\section{References}

ARDABILI HR, GARGARI BP, FARZADI L: Vitamin D supplementation has no effect on insulin resistance assessment in women with polycystic ovary syndrome and vitamin D deficiency. Nutr Res 32: 195-201, 2012. 
AZZIZ R, CARMINA E, DEWAILLY D, DIAMANTI-KANDARAKIS E, ESCOBAR-MORREALE HF, FUTTERWEIT W, JANSSEN OE, LEGRO RS, NORMAN RJ, TAYLOR AE, WITCHEL SF: Position statement: Criteria for defining polycystic ovary syndrome as a predominantly hyperandrogenic syndrome: an Androgen Excess Society Guideline. J Clin Endoc Metab 91: 4237-4245, 2006.

BONAKDARAN S, MAZLOOM KHORASANI Z, DAVACHI B, MAZLOOM KHORASANI J: The effects of calcitriol on improvement of insulin resistance, ovulation and comparison with metformin therapy in PCOS patients: a randomized placebo- controlled clinical trial. Iran J Reprod Med 10: 465-472, 2012.

BOUILLON R, VAN SCHOOR NM, GIELEN E, BOONEN S, MATHIEU C, VANDERSCHUEREN D, LIPS P: Optimal vitamin D status: a critical analysis on the basis of evidence-based medicine. Clin Endocrinol Metab 98: 1283-1304, 2013.

DIAMANTI-KANDARAKIS E, DUNAIF A: Insulin resistance and the polycystic ovary syndrome revisited: an update on mechanisms and implications. Endocr Rev 33: 981-1030, 2012.

FIGUROVÁ J, DRAVECKÁ I, JAVORSKÝ M, PETRÍKOVÁ J, LAZUROVÁ I: The effect of alfacalcidiol and metformin on metabolic disturbances in women with polycystic ovary syndrome. Horm Mol Biol Clin Investig, in press 2016.

FIGUROVÁ J, DRAVECKÁ I, JAVORSKÝ M, PETRÍKOVÁ J, LAZUROVÁ I: Prevalence of vitamin D deficiency in Slovak women with polycystic ovary syndrome and its relation to metabolic and reproductive abnormalities. Wien Klin Wochenschr 2015. DOI: 10.1007/s00508-015-0768-9.

FIROUZABADI RD, AFLATOONIAN A, MODARESSI S, SEKHAVAT L, MOHAMMAD TAHERI S: Therapeutic effects of calcium and vitamin D supplementation in women with PCOS. Complement Ther Clin Pract 18: 85-88, 2012.

HAHN S, HASELHORST U, TAN S, QUADBECK B, SCHMIDT M, ROESLER S, KIMMIG R, MANN K, JANSSEN OE: Low serum 25-hydroxyvitamin D concentrations are associated with insulin resistance and obesity in women with polycystic ovary syndrome. Exp Clin Endocrinol Diabetes 114: 577-583, 2006.

KOTSA K, YAVROPOULOU MP, ANASTASIOU O, YOVOS JG: Role of vitamin D treatment in glucose metabolism in polycystic ovary syndrome. Fertil Steril 92: 1053-1058, 2009.

LI HWR, BRERETON RE, ANDERSON RA, WALLACE AM, HO CK: Vitamin D deficiency is common and associated with metabolic risk factors in patients with polycystic ovary syndrome. Metabolism 60: 1475-1481, 2011.

MARCH WA, MOORE VM, WILLSEN KJ, PHILLIPS DI, NORMAN RJ, DAVIES MJ: The prevalence of polycystic ovary syndrome in a community sample assessed under contrasting diagnostic criteria. Hum Reprod 25 : 544-551, 2010.

RASHIDI B, HAGHOLLAHI F, SHARIAT M, ZAYERII F: The effects of calcium-vitamin D and metformin on polycystic ovary syndrome: a pilot study. Taiwan J Obstet Gynecol 48: 142-147, 2009.

SELIMOGLU H, DURAN C, KIYICI S, ERSOY C, GUCLU M, OZKAYA G, TUNCEL E, ERTURK E, IMAMOGLU S: The effect of vitamin D replacement therapy on insulin resistance and androgen levels in women with polycystic ovary syndrome. J Endocrinol Invest 33: 234-238, 2010.

TEHRANI HG, MOSTAJERAN F, SHAHSAVARI S: The effect of calcium and vitamin D supplementation on menstrual cycle, body mass index and hyperandrognism state of women with polycystic ovarian syndrom. J Res Med Sci 19: 875-880, 2014.

THYS-JACOBS S, DONOVAN D, PAPADOPOULOS A, SARREL P, BILEZIKIAN JP: Vitamin D and calcium dysregulation in the polycystic ovarian syndrome. Steroids 64: 430-535, 1999.

WEHR E, PILZ S, SCHWEIGHOFER N, GIULIANI A, KOPERA D, PIEBER TR, OBERMAYER-PIETSCH B: Association of hypovitaminosis D with metabolic disturbances in polycystic ovary syndrome. Eur $J$ Endocrinol 161: 575-582, 2009.

WEHR E, PIEBER TR, OBERMAYER- PIETSCH B: Effect of vitamin D3 treatment on glucose metabolism and menstrual frequency in polycystic ovary syndrome women: a pilot study. J Endocrinol Invest 34: 757-763, 2011. 
YILDIZHAN R, KURDOGLU M, ADALI E, KOLUSARI A, YILDIZHAN B, SAHIN HG, KAMACI M: Serum 25-hydroxyvitamin D concentrations in obese and non-obese women with polycystic ovary syndrome. Arch Gynecol Obstet 280: 559-563, 2009. 